“C 2013 IEEE. Personal use of this material is permitted. Permission from IEEE must be obtained for all other uses, in any current or future media, including reprinting/republishing this material for advertising or promotional purposes, creating new collective works, for resale or redistribution to servers or lists, or reuse of any copyrighted component of this work in other works." 


\section{Combinational Neural Logic System and Its Industrial Application on Hypoglycemia Monitoring System}

\author{
Phyo Phyo San \\ Center for Health Technologies \\ Faculty of Engineering and IT \\ University of Technology Sydney \\ Ultimo, Australia, NSW 2007 \\ Email: PhyoPhyo.San@student.uts.edu.au
}

\author{
Sai Ho Ling \\ Center for Health Technologies \\ Faculty of Engineering and IT \\ University of Technology Sydney \\ Ultimo, Australia, NSW 2007 \\ Email: Steve.Ling@uts.edu.au
}

\author{
Hung T. Nguyen \\ Center for Health Technologies \\ Faculty of Engineering and IT \\ University of Technology Sydney \\ Ultimo, Australia, NSW 2007 \\ Email: Hung.Nguyen@uts.edu.au
}

\begin{abstract}
In this paper, a combinational neural logic network (NLN) with the neural-Logic-AND, -OR and -NOT gates is applied on the development of non-invasive hypoglycemia monitoring system. It is an alarm system which measured physiological parameters of electrocardiogram (ECG) signal and determine the onset of hypoglycemia by use of proposed NLN. Due to different nature of application, conventional neural networks (NNs) with common structure may not always guarantee the optimal solution. Based on knowledge of application, the proposed NLN is designed systematically in order to incorporate the characteristics of application into the structure of proposed network. The parameter of the proposed NLN will be trained by hybrid particle swarm optimization with wavelet mutation (HPSOWM). The proposed NLN will be practically analyzed using real data sets collected from 15 children (569 data sets) with Type 1 diabetes at the Department of Health, Government of Western Australia. By using the proposed method, the detection performance is enhanced. Compared with other conventional NNs, the proposed NLN gives better performance in terms of sensitivity and specificity.
\end{abstract}

\section{INTRODUCTION}

Real-time continuous glucose monitoring has the potential to overcome diabetic complications and increase the likelihood of patients with diabetes by maintaining optimal glucose level without symptomatic hypoglycemia. In clinical practice recommendations, it has also been suggested that continuous glucose monitoring is especially useful in patients with Type 1 diabetes in order to detect hypoglycemia unawareness and/or frequent episodes of hypoglycemia.

In the past few years, glucose meters have been the method choice for measurement of blood glucose concentration for the patients with T1DM. The automatic lancet devices are used to prick the fingertip of of diabetics for taking the blood sample, which is painful as the diabetic has to measure blood glucose very frequently. Though much efforts have been made to improve with the design of glucose meters, the need for repeated measurements become the great barriers, especially for Type 1 diabetic patients who frequently need to monitor BGL as well as episodes of hypoglycemia.

The development of non-invasive continuous glucose monitoring systems is the only way for achieving painless control of blood glucose level and improving life quality of diabetes patients with better regulation of hypoglycaemia episodes. Following with the advanced iontophoresis technique, GlucoWatch G2 biographer [1] was tested and developed. However, inaccurate glucose measurements are given if the patient is moving, sweating or having rapid temperature changes. The clinical studies reported that the device performs better only at high glucose levels and does not reliable to detect low BGL of hypoglycemia [2].

Another attempt has been made to develop a wristwatchlike device named as Teledyne Sleep Sentry [3]. It is similar to GlucoWatch G2 biographer, capable of detecting falling skin temperature as well as skin resistance during sweating. However, using only skin impedance as the dominant input source become considerable factor for modeling and estimation of actual blood glucose. It cannot also overcome the issues of skin impedance such as considerable time lag and non-liner characteristics of skin in response to hypoglycemia. Due to its poor reliability, low sensitivity and specificity in detecting hypoglycemia, further improvement for the device especially the technique that is employed within the device is still needed.

Recently, advanced computational intelligence techniques such as fuzzy inference system [4] [5], fuzzy neural network estimator algorithm [6], block-based neural network [7], Bayesian neural network algorithm [8], neural network based rule discovery system [9], radial basis function neural network [10], wavelet neural network [11], and support vector machine [12] have been successfully applied for hypoglycemia detection. In neural network based hypoglycemia monitoring systems, the physiological parameters such as heart rate (HR), corrected QT interval (QTc) and skin impedance are used as the main inputs and the status of hypoglycemia is detected through the variations of those input parameters.

With the use of conventional methodologies, the obtaining sensitivity and specificity might not be able to improve further apart from the obtaining reasonable one. The need for better sensitivity and specificity will only be achieved if the different structures of neural network are designed for 
different characteristics of application. In the other words, it will be more appropriate if the structures of neural networks are employed based on the characteristics of application. Thus, in the direction of achieving truly non-invasive hypoglycemia detection system, the systematic neural network structure that will be breaking the barriers of conventional neural network become the most desirable step.

In this paper, the main contribution is devoted to the development of non-invasive hypoglycemia monitor by the use of combinational neural logic network (NLN). The proposed NLN is targeted to dedicate application and the structure of NLN systematically designed using the binary logic gates (AND, OR and NOT). In this design, the truth table and K-map are constructed depend on the knowledge of application. Due to the logic theory are used in the network design, the design of network structure becomes systematic and simpler compared to the other conventional neural network and enhance the detection performance.

In [13], which reported that hypoglycemia related to the prolongation of QT interval for both Type 1 and Type 2 diabetes patients. Since heart rate (HR) and correct QT interval (QTc) are the most common physiological parameters to be effected from hypoglycemic reaction, a combinational NLN is developed by considering correlation between physiological parameters of ECG signal and the onset of hypoglycemia. A real clinical application is given to show that the proposed NLN algorithm can successfully monitor the onset of hypoglycemic episodes.

To optimize the network parameters of NLN, a global learning algorithm called hybrid particle swarm optimization with wavelet mutation (HPSOWM) [14] is used. By using the HPSOWM, the solution quality of the training is enhanced.

The paper is organized as follows: combinational neural logic system and its training procedures by the use of HPSOWM are discussed in Section II. To show the effectiveness of the proposed NLN, an application example and results are given in Sections III and IV respectively. Finally, a conclusion is drawn in Section V.

\section{METHODS}

To develop noninvasive hypoglycemia monitoring system, the proposed combinational neural logic network in Fig. 1 is practically designed. The inputs are psychological parameters of ECG signal, the heart rate (HR) and the corrected QT interval (QTc) and the output is the presence of hypoglycemia $(h),+1$ represents hypoglycemia and -1 is non-hypoglycemia.

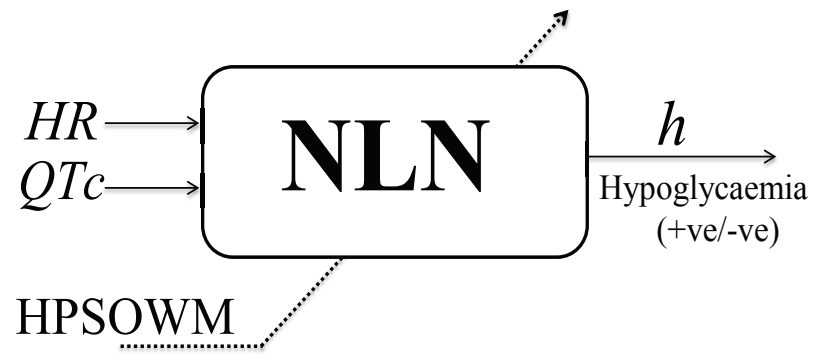

Figure 1. NLN hypoglycemia monitoring system
The proposed swarm based NLN hypoglycemia monitoring system is a type of consumer electronic device embedded in the digitized chest belt which encrypts and transmits the measured physiological parameters (heart rate and corrected QT interval) to a receiver computer using a wireless communication link. The collected data are applied to the proposed NLN algorithm in Fig. 1 to determine the level of hypoglycaemia. An alarm sound is activated when the hypoglycaemia is detected and a warning is given to the patient or physician in critical situations.

The ECG parameters under investigation involve the parameters in depolarization and repolarization stages of electrocardiography (ECG). The important points of an ECG signal in Fig. 2 are the $\mathrm{Q}$ point, $\mathrm{R}$ peak and $\mathrm{T}$ wave peak, in which $\mathrm{R}$ peak is obtained by detecting the maximum amplitude which is higher than the defined threshold level. After obtaining $\mathrm{R}$ peak, it is regarded as a reference point and the $\mathrm{Q}$ waves, and peak of the $\mathrm{T}$ wave can be found by approximating their locations at the right and left sides of R peak. In normal ECG signal the $\mathrm{Q}$ wave and peak of $\mathrm{T}$ wave are located about 120 and 300ms intervals from $\mathrm{R}$ peak [16] [17].

In Type 1 diabetic patients, prolonged QTc interval $(>440$ $\mathrm{ms}$ ) frequently occurred with spontaneous overnight hypoglycaemia when the glucose level was falling below $3.4 \mathrm{mmol} / \mathrm{l}$ [18]. A recent study [19] showed that the QTc prolongation was associated with hypoglycemia and the QTc lengthening led to $(>460 \mathrm{~ms})$ during hypoglycemia. The prevalence of prolonged QT interval (starting from Q point to the end of $\mathrm{T}$ point in Fig. 2) on the ECG signal during spontaneous hypoglycaemia in 15 children with Type 1 diabetes (aged $14.6 \pm 1.5$ years) was examined and explored for the relationships between overnight measurements of QT interval corrected for heart rate (QTc) and falling glucose level. By the use of statistical correlation analysis, it has been proven in [17] that the feature extracted ECG parameters, HR and QTc used in this study significantly increased under hypoglycemic conditions according to their respective $p$ values, HR $(\mathrm{p}<0.06)$ and QTc $(\mathrm{p}<0.001)$.

Considering a higher correlation of HR and QTc to the status of hypoglycemia, they are used as the inputs of hypoglycemia monitoring system. The design parameters of NLN are optimized by the use of HPSOWM [14]. The detail NLN algorithm, HPSOWM, the performance measurements and the formation of fitness function will discussed in the following sections.

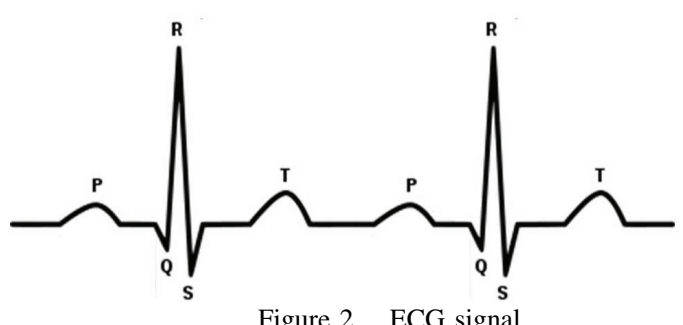

Figure 2. ECG signal 


\section{A. Design of Combinational Neural Logic Network (NLN)}

In this section, a combination neural logic system [20] consisting of rule-based logic operations and neural network will be discussed. As presented in Fig. 3, the proposed neural logic network mainly consists of (1) rule-based logic-AND, -OR and -NOT operations, (2) neural network operation and (3) combination function. In this system, the given input will firstly undergo to the rule-based logic operation which stores the boundary conditions and the properties of the logic-gates. The neural network is used to introduce the nonlinearity to the logic gates for error correction. The neural logic network output is obtained via the combination function through the use of both rule-based logic operation and the neural network outputs.

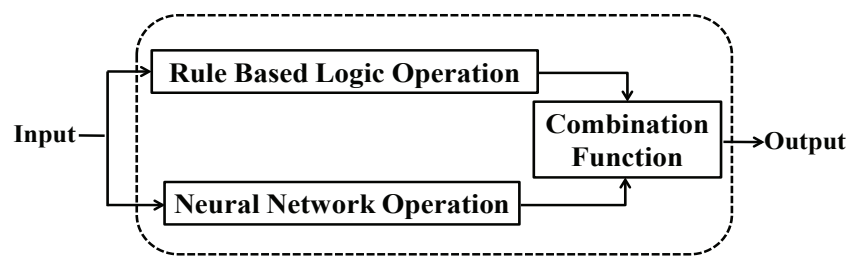

Figure 3. Block diagram of neural-logic gate

1) Rule-Based AND Gate: A two-input-single-output logic AND gate is proposed. The inputs are output lie between 0 and 1 inclusively. Referring to Fig. 3, the neural logic AND operation is defined as:

$$
x_{1}(t) \otimes x_{2}(t)=0 \vee\left(x_{1}(t)+x_{2}(t)-1\right) \in\left[\begin{array}{ll}
0 & 1
\end{array}\right]
$$

which is actually the bounded product of the input $x_{1}(t)$ and $x_{2}(t)$, where $t$ denotes the current number of input vector which is a nonzero integer. $\vee$ denotes the maximum operator. The output of the neural-logic-AND operator will be feed to the rule base which guarantees the boundary conditions and the property of a binary AND gates as shown in Table I. Throughout this paper, the neural-logic-AND operator is denoted by a $o$, e.g., $x_{1}(t)$ AND $x_{2}(t)$ is written as $x_{1}(t) \circ$ $x_{2}(t)$.

Table I

Boundary Condition AND Properties of The NEURAL Logic AND GATES

\begin{tabular}{|c|c|c|c|}
\hline$A \circ 0=0$ & $A \circ 1=A$ & $A \circ \bar{A}=0$ & $A \circ A=A$ \\
\hline $0 \circ 0=0$ & $0 \circ 1=0$ & $1 \circ 0=0$ & $1 \circ 1=1$ \\
\hline
\end{tabular}

2) Rule-Based OR Gate: A two input single output logicOR gate is presented. The inputs and output lie between 0 and 1 inclusively. Referring to Fig. 3, the logic-OR gate is defined as:

$$
x_{1}(t) \oplus x_{2}(t)=\left(x_{1}(t)+x_{2}(t)\right) \wedge 1 \in\left[\begin{array}{ll}
0 & 1
\end{array}\right]
$$

which is actually bounded sum of the inputs $x_{1}(t)$ and $x_{2}(t)$. Its operation follows the properties of a binary OR gates as shown in Table II. The symbol, • is used as the OR operator throughout this paper, e.g., $x_{1}(t)$ OR $x_{2}(t)$ is written as $x_{1}(t) \bullet$ $x_{2}(t)$.

Table II

Boundary Condition and Properties of The Neural Logic OR GATES

\begin{tabular}{|l|l|c|c|}
\hline$A \bullet 0=A$ & $A \bullet 1=1$ & $A \bullet A=A$ & $A \bullet \bar{A}=A$ \\
\hline $0 \bullet 0=0$ & $0 \bullet 1=1$ & $1 \bullet 0=1$ & $1 \bullet 1=1$ \\
\hline
\end{tabular}

3) Rule-Based NOT Gate: The logic-NOT gate has one input and one output structure and its output is defined as:

$$
y_{N O T}(t) \in\left[\begin{array}{ll}
0 & 1
\end{array}\right]=1-x(t)
$$

The logic-NOT operator is defined as 'bar', e.g.,NOT $x_{1}(t)$ is written as $\bar{x}(t)$. From (3), the characteristics of a binary logic NOT gate are retained, i.e., NOT $0=1$ and NOT $1=0$.

\section{B. Feed Forward Neural Network Architectures}

A multi layer feedforward neural network becomes the most famous due to its good approximation in any smooth and continuous nonlinear separation functions in a compact domain to arbitrary accuracy [21]. For each input has its associated

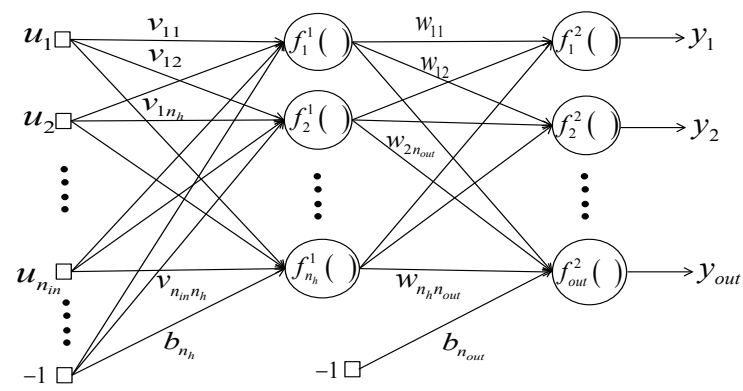

Figure 4. A three layer feedforward neural network archicture

weights and it can be modified to model synaptic learning. The input-output relationship of a fully connected feedforward neural network in Fig. 4 is calculated as follows:

$$
g_{l}=f_{l}^{2}\left(\sum_{j=1}^{n_{h}} w_{j l} f_{j}^{1}\left(\sum_{i=1}^{n_{\text {in }}} w_{i j} u_{i}-b_{j}\right)-b_{l}\right) \quad l=1,2, \ldots, n_{\text {out }}
$$

where $u_{i}, i=1,2, \ldots, n_{\text {in }}$ are the input variables, $n_{i n}$ is the number of inputs while $n_{h}$ denotes the number of hidden notes. $v_{i j}, j=1,2, \ldots, n_{h}$ is defined as the weight which is linked between the $i$ th input and $j$ th hidden nodes, $w_{j l}$ is the weight of link between the $j$ th hidden and $l$ th output nodes, $b_{j}$ and $b_{l}$ are the biases for hidden and output nodes. The total number of tuned parameters are $\left(n_{\text {in }}+1\right) \times n_{h}+\left(n_{h}+1\right) \times n_{\text {out }}$.

The transfer functions $f_{j}^{1}(\cdot)$ and $f_{l}^{2}(\cdot)$ are used as the activation function in the hidden nodes and output nodes. The following hyperbolic tangent sigmoid transfer function $\operatorname{tansig}(\theta)=\frac{1}{1+e^{-2 \theta}}-1, \in\left[\begin{array}{ll}0 & 1\end{array}\right]$ and linear transfer function pureline $(\theta)=\theta$ are commonly used in the neurons of hidden and output layers. As the neural logic network has two inputs 
and one output structure, (4) becomes as follows:

$g_{1}(t)=f_{l}^{2}\left(\sum_{j=1}^{n_{h}} w_{j 1} f_{j}^{1}\left(\sum_{i=1}^{2} v_{i j} u_{i}-b_{j}\right)-b_{1}\right), \quad i=1,2 \quad l=1$

From (1) and (5), the input-output relationship of the neural logic-AND gate can be defined as:

$$
y_{A N D}(t)=\left(0 \vee f_{A N D}\left(x_{1}(t) \otimes x_{2}(t), g_{1}(t)\right)\right) \wedge 1
$$

Consequently, the output of neural logic-OR gate can also be obtained from (2) and (5) as follows:

$$
y_{O R}(t)=\left(0 \vee f_{O R}\left(x_{1}(t) \oplus x_{2}(t), g_{1}(t)\right)\right) \wedge 1
$$

Subject to the properties of Table I and II, the symbol $\wedge$ and $V$ denote the minimum and maximum operators. The combination function $f_{A N D}(\cdot)$ and $f_{O R}(\cdot)$ for neural logicAND gate and neural logic-OR gate to be designed based on the application requirements. They satisfy the boundary conditions and exhibits the properties of the binary AND and OR gates. The total number of network parameters are calculated by $\left(n_{\text {in }}+1\right) \times n_{h}+\left(n_{h}+1\right) \times n_{\text {out }}$. The network parameters, weights and biases are $v_{i j}, w_{j l}, b_{j}$ and $b_{l}$ are optimized by hybrid particle swarm optimization with wavelet mutation (HPSOWM).

\section{Hybrid Particle Swarm Optimization with Wavelet Mutation (HPSOWM)}

In HPSOWM, a swarm $X(t)$ is constituted with the number of particles. Each particle $\mathbf{x}^{p}(t) \in X(t)$ contains $\kappa$ elements $x_{j}^{p}(t)$ at the $t$-th iteration, where $p=1,2, \ldots, \theta$ and $j=$ $1,2, \ldots, \kappa ; \theta$ denotes the number of particles in the swarm and $\kappa$ is the dimension of a particle. First, the particles of the swarm are initialized and then evaluated by a defined fitness function. The objective of HPSOWM is to minimize the fitness function (cost function) $f(X(t))$ of particles iteratively. The position $x_{j}^{p}(t)$ and velocity $v_{j}^{p}(t)$ used in HPSOWM are given as follows:

$$
\begin{aligned}
x_{j}^{p}(t) & =x_{j}^{p}(t-1)+v_{j}^{p}(t) \\
v_{j}^{p}(t) & =k \cdot\left(w \cdot v_{j}^{p}(t-1)+\varphi_{1} r_{1}\right) \cdot\left(\tilde{x}_{j}-x_{j}^{p}(t-1)\right) \\
& +\varphi_{2} r_{2}\left(\hat{x}_{j}-x_{j}^{p}(t-1)\right)
\end{aligned}
$$

where $\tilde{x}^{p}=\left[\tilde{x}_{1}^{p}, \tilde{x}_{2}^{p}, \ldots, \tilde{x}_{k}^{p}\right]$ and $\hat{\mathbf{x}}=\left[\hat{x_{1}} \hat{x_{2}}, \ldots \hat{x_{\kappa}}\right], j=1,2, \ldots, \kappa$. The best previous position of a particle is recorded and represented as $\tilde{x}$; the position of best particle among all the particles is represented as $\hat{x} ; w$ is an inertia weight factor; $r_{1}$ and $r_{2}$ are acceleration constants which return a uniform random number in the range of $[0,1] ; w$ is inertia weight factor and $k$ is a constriction factor which detail derivation is discussed [14].

$$
\bar{x}_{j}^{p}(t)=\left\{\begin{array}{cc}
x_{j}^{p}(t)+\sigma \times\left(\rho_{\max }^{j}-x_{j}^{p}(t)\right) & , \sigma>0 \\
x_{j}^{p}(t)+\sigma \times\left(x_{j}^{p}(t)-\rho_{\min }^{j}\right) & , \sigma<0
\end{array}\right.
$$

where $j \in 1,2, \ldots, \kappa$ and $\kappa$ denotes the dimension of particles. The value of $\sigma$ is governed by Morlet wavelet function as presented in [14].

\section{Fitness Function and Training}

To determine the performance of proposed detection system, Sensitivity, $\xi=\frac{N_{T P}}{N_{T P}+N_{F N}}$ and Specificity $\eta=\frac{N_{T N}}{N_{T N}+N_{F P}}$ in [22] are introduced in which $N_{T P}$ is the number of true positive, $N_{F N}$ is number of false negative, $N_{F P}$ is number of false positive and $N_{T N}$ is number of true negative. These values are within 0 to 1 . The objective of proposed detection system is to maximize fitness function $f(\xi, \eta)$ in (10) which is equivalent to maximization of the sensitivity and the specificity. In order analyze the performance of proposed detection system, the fitness function is defined as:

$$
f(\xi, \eta)=\xi+\bar{\eta}
$$

where $\xi$ and $\eta$ are sensitivity and specificity. In order to analyze sensitivity, $\eta_{\max }$ is defined from 0 to 1

$$
\bar{\eta}= \begin{cases}1 & \text { if } \eta \geq \eta_{\max } \\ 1-\left(\frac{\eta_{\max }-\eta}{\eta_{\max }}\right) & \text { if } \eta<\eta_{\max }\end{cases}
$$

where $\eta_{\max }$ is a upper limit of the specificity and it is used to fix the region of specificity from 0 to 1 and find the optimal sensitivity in this region. To find the optimal parameters of VTWNN, the parameters of the HPSOWM are selected as: swarm size $\theta=50$, constant value $c_{1}$ and $c_{2}=2.05$, maximum velocity $v_{\max }=0.3$, probability of mutation $\mu_{m}=0.7$, the shape parameter of wavelet mutation $\zeta_{w m}=2$, the constant value $g$ of wavelet mutation $=10000$ and the number of iteration $T=2000$.

\section{APPLICATION EXAMPLE}

To illustrate the design procedure and the merits of proposed approach, the proposed combinational neural-logic system is employed to perform detection of hypoglycemia. A truth table is firstly set up which governs the relations among some linguistic states i.e., $\mathrm{H}$ for high and $\mathrm{L}$ for low. The $\mathrm{H}$ and L in binary logic systems refer to crisp 1 and 0 respectively. The NLN system in Fig. 1, the physiological parameters of ECG signal such as HR and QTc are used as the inputs while $\mathrm{y}$ is used as the system output. For variable HR and QTc and their two linguistic states, $\mathrm{H}$ and $\mathrm{L}$ given in the truth table, Table III. These two linguistics states divides the input into two subregions, 0 to 1 .

Table III

Truth Table for Hypoglycemia Monitoring System

\begin{tabular}{|c|c|c|}
\hline HR & QTc & y \\
\hline H & H & H \\
\hline H & L & L \\
\hline L & H & L \\
\hline L & L & L \\
\hline
\end{tabular}

Depend on the characteristics of applications, for a value of input $\mathrm{HR}$, it is takes as logic condition 1 (H-High) if normalized $H R \in[1.01 \sim 2.575]$ whereas 0 (L-Low) if $H R \in$ 
[0.479 1.0019]. The same condition applied for QTc, it is defined as 1 (H-High) if $Q T c \in[1.034 \sim 1.41]$ else 0 (L-Low) if normalized $Q T c \in[0.7 \sim 1.033]$. For both HR and QTc, the values which are out of theses boundary conditions take as don't care condition (X). Picking up the first row in Table III as an example, it can be interpreted that if $\mathrm{HR}$ is " $\mathrm{H}$ " and QTc is " $\mathrm{H}$ ", then $y$ is "H". These rules are determined based on human knowledge about the problem to be handled. Based on the own characteristics of this clinical application,

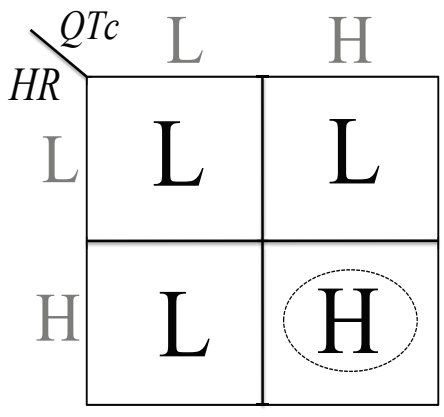

Figure 5. K map

the proposed NLN is designed to exactly follow the properties of logic-AND gates in Table I. The status of hypoglycemia is mainly occurred when the HR and QTc are higher, i.e., if HR is " $\mathrm{H}$ " and QTc is " $\mathrm{H}$ ", then $y$ is " $\mathrm{H}$ " in Table .III. For instances, the other conditions in Table .III, such as HR is " $\mathrm{H}$ " and QTc is "L", HR is "L" and QTc is " $H$ " and HR is "L" and QTc is "L", it is considered as the occurrence of hypoglycemia is not as distinct as the condition when HR and QT are "H" and the $y$ is defined as "L".

Based on the Table III and K-Map in Fig. 5, the following logic expression governed by the logic-AND gate (Table I) is obtained as the output of NLN system:

$$
y=H R \circ Q T c
$$

The combination function in Fig. 3 is selected as 'tansig', i.e., $f_{A N D}\left(v_{1}, v_{2}\right)=$ tansig $\left(v_{1}+v_{2}\right)$ in order to map the inputs to be $\left[\begin{array}{ll}-1 & 1\end{array}\right]$. Other functions could be replaced depend on the application. In this application, they are chosen experimentally to give a satisfactory result. Based on the output of NLN, $y$ in (11), the status of hypoglycemia $h$ in Fig. 1 is expressed as:

$$
h=\left\{\begin{array}{cc}
+1, & y \geq 0 \\
-1, & y<0 .
\end{array}\right.
$$

This application illustrates the idea of designing a two-inputsingle output combinational neural logic system. The idea can readily be extended to design a multiple-input-multiple-output combinational neural-logic system. It should be aware that the design of NLN, such as the construction of truth table and K-map in Table III and Fig. 5, the definition of combination function in Fig. 3, are largely depended on the characteristics of application.

\section{RESULT AND DISCUSSION}

In this group of study, all 15 patients (children with ages $14.6 \pm 1.5$ years) were Type 1 diabetes patients and they underwent for overnight electrocardiogram (ECG) and glucose monitoring at the Princess Margaret Hospital for Children in Perth, Australia. For each patient, all the data were collected between 360 minutes to 480 minutes and approximately 35-40 data points were collected for a sampling period of between 5 and 10 minutes.

In this clinical application, BGL $<3.3 \mathrm{mmol} / l$ was considered hypoglycemic episodes. The relationships between overnight measurements of QT interval corrected for heart rate (QTc) and falling BGL were discovered by the use of statistical correlation analysis. The overall data set of 15 children with Type 1 diabetes [17] were considered in this study because of their significant increment in HR, QTc in response to episodes of hypoglycemia by giving $\mathrm{p}$ values as (1.033 \pm 0.242 vs. $1.082 \pm 0.242 \pm<0.06)$ for HR and (1.031 \pm 0.086 vs. $1.060 \pm 0.084<0.001)$ for QTc. A training set $(5$ patients with 184 data points), a validation set (5 patients with 192 data points) and a testing set (5 patients with 153 data points) are randomly selected.

Four comparison approaches such as neural logic network (NLN), wavelet neural network (WNN), feedforward neural network (FFNN) and statical multi regression (MR) are compared and analyzed. In this clinical application, the sensitivity which mainly identifies actual hypoglycemic episodes is more important than specificity. To analyze this, the fitness function (10) was designed and find the optimized sensitivity while the specificity was kept at $\eta_{\max }=40 \%$.

Table IV

Mean Value of Training, Validation and Testing Results: Set MAXIMUM SPECIFICITY, $\eta_{\max }=40 \%$

\begin{tabular}{l|cc|cc|ccc}
\hline Method & \multicolumn{2}{|c|}{ Training(\%) } & \multicolumn{2}{|c|}{ Validation(\%) } & \multicolumn{3}{c}{ Testing(\%) } \\
& Sen & Spec & Sen & Spec & Sen & Spec & $\gamma$ \\
\hline \hline NLN & 90.23 & 41.28 & 86.81 & 40.00 & 74.93 & 53.83 & 66.49 \\
\hline WNN & 83.82 & 40.66 & 80.00 & 41.75 & 70.93 & 50.36 & 62.70 \\
\hline FFNN & 81.06 & 41.26 & 76.41 & 41.44 & 65.93 & 50.09 & 59.59 \\
\hline MR & 85.76 & 40.89 & 73.28 & 40.00 & 63.37 & 59.74 & 61.92 \\
\hline
\end{tabular}

Table V

Best Testing Result FOR HyPOGLYCEMia DETECTION With DIFFERENT APPROACHES: SET MAXIMUM SPECIFICITY, $\eta_{\max }=40 \%$

\begin{tabular}{l|c|c|c}
\hline Method & $\operatorname{Sens}(\%)$ & $\operatorname{Spec}(\%)$ & $\gamma$ \\
\hline \hline NLN & $76.74 \%$ & $54.55 \%$ & $67.86 \%$ \\
\hline WNN & $72.09 \%$ & $50.91 \%$ & $63.61 \%$ \\
\hline FFNN & $67.44 \%$ & $54.55 \%$ & $62.28 \%$ \\
\hline MR & $62.79 \%$ & $60.91 \%$ & $62.04 \%$ \\
\hline
\end{tabular}

At the defined cut-off point $\eta_{\max }=40 \%$, the average (mean) sensitivity and specificity of training, validation and testing results over 20 runs are calculated as presented in Table IV. The mean testing result of proposed NLN was found to be 
satisfactory with sensitivity and specificity, (74.93 and 53.83\%) compared to the other conventional classifiers such as WNN (72.09 and 50.91\%), FFNN (67.44 and 54.55\%) and MR (62.79 and $60.91 \%)$.

For performance evaluation, gamma $(\gamma)$ analysis $(\gamma=\theta \xi+$ $(1-\theta) \eta, \theta$ is in the range of 0.1 to 1$)$ is defined in this studies. To meet with the minimum requirement of proposed system (sensitivity $\geq 60 \%$ and specificity $\geq 40 \%$ ), $\theta$ was set to 0.6 (which is equivalent to sensitivity $60 \%$ ). By substituting $\theta$, the $\gamma$ analysis becomes $\gamma=0.6 \xi+0.4 \eta$. The obtained mean sensitivity and specificity were evaluated in terms of $\gamma$ analysis. In Table IV, it can also be found that the proposed NLN gives better $\gamma$ value at $66.49 \%$.

In terms of the best performance, the proposed NLN achieves better sensitivity and specificity value of $76.74 \%$ and $54.55 \%$ whereas WNN, FFNN have MR have $72.09 \%$ and $50.91 \%, 67.44 \%$ and $54.55 \%, 62.79 \%$ and $60.91 \%$ respectively. In summary, the proposed NLN based hypoglycemia monitoring system is effective with better sensitivity and specificity.

\section{CONCLUSIONS}

A combinational neural logic network is developed for hypoglycemia monitoring system based on the changes in physiological parameters of ECG signal during hypoglycemia and non-hypoglycemia reactions. Traditionally, neural networks with the same structure were applied to handle every applications and may not give the optimal solution due to different characteristics of applications. In this application, the status of hypoglycemia is detected through the neural logic-AND gates which worked as the basic building block of proposed NLN. With the help of a truth table and K-map, the proposed NLN is designed to systematically incorporate the characteristics of application to the NLN structure. The results in Section IV indicate that the hypoglycemia episodes in T1DM children can be efficiently detected non-invasively and continuously with better sensitivity $(76.74 \%)$ and specificity $(54.55 \%)$.

\section{ACKNOWLEDGMENT}

The authors would like to thank Dr. Nejhdeh Ghevondian, and Assoc. Prof. Timothy Jones for their contribution. This works was supported by a grant from Juvenile Diabetes Research Foundation.

\section{REFERENCES}

[1] M. J. Tierney, J. A. Tamada, R. O. Potts and L. Jovanovic, "Clinical evaluation of the GlucoWatch biographer: a continual, non-invasive glucose monitor for patients with diabetes", Biosens Bioelectron, vol. 16, pp. 621-629, 2001.

[2] E. Tsalikian, C. Kollman and N. Mauras, "GlucoWatch G2 Biographer alarm reliability during hypoglycemia in children", Diabetes Technology and Therapeutics, vol. 6, no. 5, pp. 559-566, 2004.

[3] K. A. Hansen and S. C. Duck, "Teledyne sleep sentry: evaluation in pediatric patients for detection of nocturnal hypoglycemia", Diabetes Care, vol. 6, pp. 597-600, 1983.

[4] S. H. Ling and H.T. Nguyen, "Natural occurrence of nocturnal hypoglycemia detection using hybrid particle swarm optimized fuzzy reasoning model”, Artificial Intell. in Medicine, vol. 55, pp. 177-184, 2012.
[5] S. H. Ling, H. T. Nguyen and F. H. F Leung, "Hypoglycemia detection using fuzzy inference system with genetic algorithm", Proceeding of the 2011 IEEE International Conference on Fuzzy Systems, Taiwan, June, 2011, pp. 2225-2231.

[6] N. Ghevondian and H. T. Nguyen, "A novel fuzzy neural network estimator for predicting hypoglycaemia in insulin-induced subjects", Proceedings of the 23rd Annual International Conference of the IEEE Engineering in Medicine and Biology Society, Turkey, Oct. 2001, pp. 1657-1660.

[7] P. P. San, S. H. Ling, and H. T. Nguyen, "Industrial application of evolvable block-based neural network to hypoglycemia monitoring system", IEEE Trans. Industrial Electronics. (in-press)

[8] H. T. Nguyen, N. Ghevondian, S. T. Nguyen and T. W. Jones, "Detection of hypoglycemic episodes in children with type 1 diabetes using an optimal Bayesian neural network algorithm", Proceedings of the International Conference of IEEE Engineering in Medicine and Biology Society, Lyon, Aug. 2007, pp. 3140-3143.

[9] K. Y. Chan, S. H. Ling, T. S. Dillion, and H. T. Nguyen, "Diagnosis of hypoglycemic episodes using a neural network based rule discovery system", Expect system and Applications, vol. 38, no. 8, pp. 9799-9808, 2011.

[10] P. P. San, S. H. Ling, and H. T. Nguyen, "Hybrid particle swarm optimization based normalized radial basis function neural network for hypoglycemia detection", in Proc. International Joint Conference on Neural Networks 2012, Australia, June 2012, pp. 2718-2723.

[11] P. P. San, S. H. Ling, and H. T. Nguyen, "Hybrid PSO-based variable translation wavelet neural network and its application to hypoglycemia detection system", Neural Computing and Applications. (in-press)

[12] N. Nuryani, S. H. Ling, and H. T. Nguyen, "Electrocardiographic signals and swarm based support vector machine for hypoglycemia detection", Annals of Biomedical Engineering, vol. 40, no. 4, pp. 934-945, 2012.

[13] S. P. Lee, L. Yeoh and N. D. Harris, "Influence of autonomic neuropathy on QTc interval lengthening during hypoglycemia in type 1 diabetes", Diabetes, vol. 53, pp. 1535-1542, 2004.

[14] S. H. Ling, H. H. C. Iu, K.Y. Chan, H.K. Lam, C.W. Yeung and F.H.F Leung, "Hybrid particle swarm optimization with wavelet mutation and its industrial applications", IEEE Trans. Sys., Man, and Cybernetics, Part $B$, vol. 38, no. 3, pp. 743-763, 2008.

[15] S. S. Haykin, Neural networks : a comprehensive foundation, Prentice Hall, Upper Saddle River, NJ, 1999.

[16] S. H. Ling and H. T. Nguyen, "Genetic-Algorithm-Based Multiple Regression With Fuzzy Inference System for Detection of Nocturnal Hypoglycemic Episodes", IEEE Transactions on Information Technology in Biomedicine, vol. 15, no. 2, pp. 308-315, 2011.

[17] H. T. Nguyen, N. Ghevondian and T. W. Jones, "Detection of nocturnal hypoglycemic episodes (natural occurrence) in children with Type 1 diabetes using an optimal Bayesian neural network algorithm", Proceeding of the 30th Annual International Conference of the IEEE Engineering in Medicine and Biology Society, Canada, Aug. 2008, pp. 1311-1314.

[18] N. P. Murphy, M. E. Ford-Adams and K. K. Ong, "Prolonged cardiac repolarisation during spontaneous nocturnal hypoglycaemia in children and adolescents with type 1 diabetes", Diabetologia, vol. 47, pp. 19401947, 2004.

[19] L. L. Nguyen, S. Su and Hung T. Nguyen, "Identification of Hypoglycemia and Hyperglycemia in Type 1 Diabetic Patients Using ECG Parameters", Proceeding of the 34th Annual International Conference of the IEEE Engineering in Medicine and Biology Society, California, USA, Aug. 2012, pp. 2716-2719.

[20] H. K. Lam and F. H. F. Leung, "Design and training for combinational neural-logic systems", IEEE Transactions on Industrial Electronics, vol. 54, no. 1, pp. 612-619, 2007.

[21] B. Windrow and M. A. Lehr, "30 years of adaptive neural networks: Perception, madaline and backpropagation", Proceeding of The IEEE, vol. 78, no. 9, pp. 1415-1442, 1990.

[22] D. G. Altman and J. M. Bland, "Statistics Notes: Diagnostic tests 1: sensitivity and specificity", Clinical Chemistry, pp. 1552-1552, 1994. 